\title{
Global attractivity of almost periodic solutions for competitive Lotka-Volterra diffusion system ${ }^{* \dagger}$
}

\author{
Ahmadjan Muhammadhaji, Zhidong Teng; Mehbuba Rehim \\ College of Mathematics and Systems Science, Xinjiang University \\ Xinjiang, Urumqi 830046, P.R.China
}

Received: 23-12-2012 / Accepted: 23-01-2013/ Accepted for publication in AMV

\begin{abstract}
In this paper, two competitive Lotka-Volterra populations in the two-patch-system with diffusion are considered. Each of the two spiecies can diffuse indepently and discretely between its in intrapatch and interpatch. By means of constructing Liapunov function, under moderate condition, the system has a unique almost periodic solution and which is asymptotically stable and globally attractive .

Key words: Lotka-Volterra competitive system; diffusion; almost periodic solution; asymptotically stability; global attractivity
\end{abstract}

\section{Introduction}

Diffusion is a ubiquitous phenomenon in the real world. It is population pressure due to the mutual interference between the individuals, describing the migration of species to avoid crowds. It is important for us to understand the dynamics of populations of nature, and the basic and important studied questions for the dynamics of populations are the persistence, permanence and extinction of species, global stability of systems and the existence of positive periodic solutions, positive almost periodic solutions and asymptotically periodic solutions, etc. Recently, many scholars have paid attention to the non-autonomous Lotka-Volterra population models with diffusion. There exists an extensive literature concerning the study of global stability and the existence of positive periodic solutions, positive almost periodic

\footnotetext{
*2000 Mathematics Subject Classification. 34K20; 34D23; 34D45; 34D05; 34K14.

${ }^{\dagger}$ This work was supported by the National Natural Science Foundation of China (Grant Nos. 11271312, 11261058), the China Postdoctoral Science Foundation (Grant Nos. 20110491750), the Natural Science Foundation of Xinjiang (Grant Nos. 2011211B08).

${ }_{\ddagger}^{\ddagger}$ Corresponding author. Tel/Fax: +86 991 8585505, Email: zhidong@xju.edu.cn(Z. Teng), ahmatjanam@yahoo.com.cn(A. Muhammadhaji).
} 
solutions and asymptotically periodic solutions of Lotka-Volterra system with diffusion and periodic parameters, see the monographs [1-3,5-19] and the references cited therein.

In [12], the authors studied the following nonautonomous Lotka-Volterra almost periodic cooperative systems with diffusion

$$
\left\{\begin{array}{l}
\dot{x}_{1}=x_{1}\left[r_{1}(t)-a_{11}(t) x_{1}+a_{12}(t) y_{1}\right]+D_{1}(t)\left(x_{2}-x_{1}\right), \\
\dot{y}_{1}=y_{1}\left[r_{2}(t)+a_{21}(t) x_{1}-a_{22}(t) y_{1}\right]+D_{2}(t)\left(y_{2}-y_{1}\right), \\
\dot{x}_{2}=x_{2}\left[s_{1}(t)-b_{11}(t) x_{2}+b_{12}(t) y_{2}\right]+D_{1}(t)\left(x_{1}-x_{2}\right) \\
\dot{y}_{2}=y_{2}\left[s_{2}(t)+b_{21}(t) x_{2}-b_{22}(t) y_{2}\right]+D_{2}(t)\left(y_{1}-y_{2}\right),
\end{array}\right.
$$

By means of constructing Liapunov function and under appropriate conditions, the sufficient conditions on the existence of a unique almost periodic solution and its global asymptotic stability are established for system (1.1). Based on the system (1.1), in [13], the authors generalized almost periodic system (1.1) into asymptotically periodic systems, under suitable conditions, the authors obtained that asymptotically periodic systems have a unique solution which is globally asymptotically stable.

In [11], the authors studied the following nonautonomous Lotka-Volterra periodic competitive systems with diffusion

$$
\left\{\begin{array}{l}
\dot{x}_{1}=x_{1}\left[r_{1}(t)-a_{11}(t) x_{1}-a_{12}(t) y_{1}\right]+D_{1}(t)\left(x_{2}-x_{1}\right), \\
\dot{y}_{1}=y_{1}\left[r_{2}(t)-a_{21}(t) x_{1}-a_{22}(t) y_{1}\right]+D_{2}(t)\left(y_{2}-y_{1}\right), \\
\dot{x}_{2}=x_{2}\left[s_{1}(t)-b_{11}(t) x_{2}-b_{12}(t) y_{2}\right]+D_{1}(t)\left(x_{1}-x_{2}\right), \\
\dot{y}_{2}=y_{2}\left[s_{2}(t)-b_{21}(t) x_{2}-b_{22}(t) y_{2}\right]+D_{2}(t)\left(y_{1}-y_{2}\right),
\end{array}\right.
$$

By using of Brouwer fixed point theorem and constructing a suitable Liapunov function, under some appropriate conditions, the authors obtained that the system has a unique periodic solution which is globally stable.

Motivated by the works [12] and [13] of Wei and Wang, by using of Liapunov method used in $[12,13,14]$, we generalize system (1.2) into almost periodic system, under suitable conditions, we obtain that the system has a unique almost periodic solution which is asymptotically stable and globally attractive.

The organization of this paper is as follows. In the next section we will present some basic assumptions, notations and Lemmas. In section 3, conditions for the almost periodic solution and asymptotic stability are considered. In section 4, conditions for the global attractivity are considered. In the final section, one example is given to illustrate that our main results are applicable. 


\section{Preliminaries}

In system (1.2), we have that $x_{1}(t), y_{1}(t)$ are the density of two competitive species at time $\mathrm{t}$ at the first patch, $x_{2}(t), y_{2}(t)$ are the density of two competitive species at time t at the second patch, $r_{i}(t)$ and $s_{i}(t)$ are intrinsic growth rate of two competitive species at the first and second patch respectively, $a_{i i}(t)$ and $b_{i i}(t)$ are intrapatch restriction density of each species in two-patch-system, $a_{i j}(t), b_{i j}(t)(i \neq j)$ are competitive coefficients between two species, $D_{i}(t)$ are diffusion coefficients. In this paper, we always assume that system (1.2) satisfies the following assumption

$\left(H_{1}\right) r_{i}(t), s_{i}(t), a_{i j}(t), b_{i j}(t)$ and $D_{i}(t)$ are nonnegative continuous bounded almost periodic functions $(i, j=1,2)$.

From the viewpoint of mathematical biology, in this paper for system (1.2) we only consider the solution with the following initial condition

$$
x_{i}(t)=\phi_{i}(t), y_{i}(t)=\varphi_{i}(t) \quad \text { for all } \quad t \in[0,+\infty), i=1,2
$$

where $\phi_{i}(t), \varphi_{i}(t)(i=1,2)$ are nonnegative continuous functions defined on $[0,+\infty)$ satisfying $\phi_{i}(0)>0, \varphi_{i}(0)>0(i=1,2)$.

For a continuous and bounded function $f(t)$ defined on $[0,+\infty)$, we define $f^{L}=$ $\inf _{t \in[0,+\infty)}\{f(t)\}$ and $f^{M}=\sup _{t \in[0,+\infty)}\{f(t)\}$.

Now, we present some useful lemmas.

Lemma 2.1. [11] $R_{+0}^{4}=\left\{\left(x_{1}, y_{1}, x_{2}, y_{2}\right) \in R^{4} \mid x_{i} \geq 0, y_{i} \geq 0, \quad(i=1,2)\right\}$ is the positive invariance set with respect to the system (1.2).

Lemma 2.2. [11] If the following inequalities hold

$$
D_{1}^{M}<r_{1}^{L}, \quad D_{2}^{M}<r_{2}^{L}, \quad D_{1}^{M}<s_{1}^{L}, \quad D_{2}^{M}<s_{2}^{L},
$$

then there exists a compact region which has a positive distance from the coordinate hyperplane and it attracts all the solutions of the system (1.2) with positive initial values.

Lemma 2.3. [12] Let $D$ be an open set of $R_{+}^{4}$, function $V(t, x, y)$ be defined on the region $R_{+} \times D \times D$ or $R_{+} \times R_{+}^{4} \times R_{+}^{4}$, it satisfies that:

(i) $a(\|x-y\|) \leq V(t, x, y) \leq b(\|x-y\|)$, where $a(r)$ and $b(r)$ are continuous increasing positive functions;

(ii) ||$V\left(t, x_{1}, y_{1}\right)-V\left(t, x_{2}, y_{2}\right) \| \leq k\left(\left\|x_{1}-x_{2}\right\|+\left\|y_{1}-y_{2}\right\|\right), k>0$ is a constant;

(iii) $V^{\prime}(t, x, y) \leq c V(t, x, y)$, where $c>0$ is a constant.

Further, let the solution of system (1.2) lie in compact set $\Omega$ for all $t \geq t_{0}>0, \Omega \in D$, 
then system (1.2) has a unique almost periodic solution $z(t)$ in $D, z(t)$ lies in $\Omega$, and it is uniformly asymptotically stable.

Lemma 2.4. [4] Let $f$ be a nonnegative function defined on $[0, \infty)$ such that $f$ is integrable on $[0, \infty)$ and uniformly continuous on $[0, \infty)$. Then $\lim _{t \rightarrow \infty} f(t)=0$.

F.Wei et al. obtained in [11] that the system (1.2) had a bounded closed and convex set

$\Omega=\left\{z: z \in R_{+}^{4}, S(z) \leq \beta, x_{i}^{L} \leq x_{1} \leq x_{i}^{M}, y_{i}^{L} \leq y_{1} \leq y_{i}^{M}(i=1,2), R_{+}=[0,+\infty)\right\}$.

where $S(z), \beta, x_{i}^{L}, x_{i}^{M}, y_{i}^{L}, y_{i}^{M}$ are defined in [11, Theorem 3.1 and Theorem 4.1].

We discuss system (1.2) in $\Omega$. In order to obtain almost periodic solution and asymptotic stability of system (1.2) we introduce the following adjoint system (2.1) of system (1.2)

$$
\left\{\begin{array}{l}
\dot{x}_{1}=x_{1}\left[r_{1}(t)-a_{11}(t) x_{1}-a_{12}(t) y_{1}\right]+D_{1}(t)\left(x_{2}-x_{1}\right), \\
\dot{y}_{1}=y_{1}\left[r_{2}(t)-a_{21}(t) x_{1}-a_{22}(t) y_{1}\right]+D_{2}(t)\left(y_{2}-y_{1}\right), \\
\dot{x}_{2}=x_{2}\left[s_{1}(t)-b_{11}(t) x_{2}-b_{12}(t) y_{2}\right]+D_{1}(t)\left(x_{1}-x_{2}\right), \\
\dot{y}_{2}=y_{2}\left[s_{2}(t)-b_{21}(t) x_{2}-b_{22}(t) y_{2}\right]+D_{2}(t)\left(y_{1}-y_{2}\right), \\
\dot{\tilde{x}}_{1}=\tilde{x}_{1}\left[r_{1}(t)-a_{11}(t) \tilde{x}_{1}-a_{12}(t) \tilde{y}_{1}\right]+D_{1}(t)\left(\tilde{x}_{2}-\tilde{x}_{1}\right), \\
\dot{\tilde{y}}_{1}=\tilde{y}_{1}\left[r_{2}(t)-a_{21}(t) \tilde{x}_{1}-a_{22}(t) \tilde{y}_{1}\right]+D_{2}(t)\left(\tilde{y}_{2}-\tilde{y}_{1}\right) \\
\dot{\tilde{x}}_{2}=\tilde{x}_{2}\left[s_{1}(t)-b_{11}(t) \tilde{x}_{2}-b_{12}(t) \tilde{y}_{2}\right]+D_{1}(t)\left(\tilde{x}_{1}-\tilde{x}_{2}\right) \\
\dot{\tilde{y}}_{2}=\tilde{y}_{2}\left[s_{2}(t)-b_{21}(t) \tilde{x}_{2}-b_{22}(t) \tilde{y}_{2}\right]+D_{2}(t)\left(\tilde{y}_{1}-\tilde{y}_{2}\right) .
\end{array}\right.
$$

Such adjoint system can be found in $[7,12,13]$.

\section{Almost periodic solution and asymptotic sta- bility}

In this section, we will derive some sufficient conditions for the existence of almost periodic solution and its asymptotic stability of system (1.2).

Theorem 3.1. If the conditions of $\left(H_{1}\right)$, Lemma 2.2 and Lemma 2.3 hold, and further assume that system (1.2) satisfies

$$
a_{11}^{L}+a_{21}^{L}>\frac{D_{1}^{M}}{x_{2}^{L}}, \quad b_{11}^{L}+b_{21}^{L}>\frac{D_{1}^{M}}{x_{1}^{L}}, \quad a_{12}^{L}+a_{22}^{L}>\frac{D_{2}^{M}}{y_{2}^{L}}, \quad b_{12}^{L}+b_{22}^{L}>\frac{D_{2}^{M}}{y_{1}^{L}} .
$$

then system (1.2) has a unique almost periodic solution which is uniformly asymptotically stable. 
Proof. Let $z(t)=\left(x_{1}(t), x_{2}(t), y_{1}(t), y_{2}(t)\right)$ be any solution of system (1.2), from the attraction of $\Omega$, we discuss our problem in $\Omega$, and denote

$$
Z(t)=\left(X_{1}(t), X_{2}(t), Y_{1}(t), Y_{2}(t)\right)
$$

We consider the adjoint system (2.1) of system (1.2), let

$$
X_{i}(t)=\ln x_{i}(t), \quad Y_{i}(t)=\ln y_{i}(t), \quad \tilde{X}_{i}(t)=\ln \tilde{x}_{i}(t), \quad \tilde{Y}_{i}(t)=\ln \tilde{y}_{i}(t)(i=1,2),
$$

where $x_{1}(t), y_{i}(t), \tilde{x}_{i}(t),, \tilde{y}_{i}(t)(i=1,2)$ are the solutions of adjoint system (2.1) on $\Omega \times \Omega$.

Define Liapunov function:

$$
V(t)=V(t, Z(t), \tilde{Z}(t))=\sum_{i=1}^{2}\left|X_{i}(t)-\tilde{X}_{i}(t)\right|+\sum_{i=1}^{2}\left|Y_{i}(t)-\tilde{Y}_{i}(t)\right|
$$

Taking

$$
a(r)=b(r)=\sum_{i=1}^{2}\left|X_{i}(t)-\tilde{X}_{i}(t)\right|+\sum_{i=1}^{2}\left|Y_{i}(t)-\tilde{Y}_{i}(t)\right|
$$

and $a(r), b(r)$ are continuous increasing positive functions, then $V(t)$ satisfies the condition (i) of Lemma 2.3. Again from

$$
\begin{aligned}
& \sum_{i=1}^{2}\left|X_{i 1}(t)-\tilde{X}_{i 1}(t)\right|+\sum_{i=1}^{2}\left|Y_{i 1}(t)-\tilde{Y}_{i 1}(t)\right|-\left(\sum_{i=1}^{2}\left|X_{i 2}(t)-\tilde{X}_{i 2}(t)\right|+\sum_{i=1}^{2}\left|Y_{i 2}(t)-\tilde{Y}_{i 2}(t)\right|\right) \\
& \leq \sum_{i=1}^{2}\left|X_{i 1}(t)-X_{i 2}(t)\right|+\sum_{i=1}^{2}\left|Y_{i 1}(t)-Y_{i 2}(t)\right|+\sum_{i=1}^{2}\left|\tilde{X}_{i 1}(t)-\tilde{X}_{i 2}(t)\right|+\sum_{i=1}^{2}\left|\tilde{Y}_{i 1}(t)-\tilde{Y}_{i 2}(t)\right|
\end{aligned}
$$

hence $V(t)$ satisfies the condition (ii) of Lemma 2.3. To check the condition (iii) of Lemma 2.3, we need to calculate upper-right derivative of system (2.1), for convenience of statements we denote

$$
A_{i}=\operatorname{sign}\left(\left|X_{i}(t)-\tilde{X}_{i}(t)\right|\right), B_{i}=\operatorname{sign}\left(\left|Y_{i}(t)-\tilde{Y}_{i}(t)\right|\right),(i=1,2)
$$




$$
\begin{aligned}
D^{+} V(t)= & D^{+}\left(\sum_{i=1}^{2}\left|X_{i}(t)-\tilde{X}_{i}(t)\right|+\sum_{i=1}^{2}\left|Y_{i}(t)-\tilde{Y}_{i}(t)\right|\right) \\
= & \sum_{i=1}^{2} D^{+}\left(\left|X_{i}(t)-\tilde{X}_{i}(t)\right|\right)+\sum_{i=1}^{2} D^{+}\left(\left|Y_{i}(t)-\tilde{Y}_{i}(t)\right|\right) \\
= & \sum_{i=1}^{2} A_{i}\left(\dot{X}_{i}(t)-\dot{\tilde{X}}_{i}(t)\right)+\sum_{i=1}^{2} B_{i}\left(\dot{Y}_{i}(t)-\dot{\tilde{Y}}_{i}(t)\right) \\
= & \sum_{i=1}^{2} A_{i}\left(\frac{\dot{x}_{i}(t)}{x_{i}(t)}-\frac{\dot{x}_{i}(t)}{\tilde{x}_{i}(t)}\right)+\sum_{i=1}^{2} B_{i}\left(\frac{\dot{y}_{i}(t)}{y_{i}(t)}-\frac{\dot{\tilde{y}}_{i}(t)}{\tilde{y}_{i}(t)}\right) \\
= & -A_{1} a_{11}(t)\left(x_{1}-\tilde{x}_{1}\right)-A_{1} a_{12}(t)\left(y_{1}-\tilde{y}_{1}\right)+A_{1} D_{1}(t)\left(\frac{x_{2}}{x_{1}}-\frac{\tilde{x}_{2}}{\tilde{x}_{1}}\right) \\
& -A_{2} b_{11}(t)\left(x_{2}-\tilde{x}_{2}\right)-A_{2} b_{12}(t)\left(y_{2}-\tilde{y}_{2}\right)+A_{2} D_{1}(t)\left(\frac{x_{1}}{x_{2}}-\frac{\tilde{x}_{1}}{\tilde{x}_{2}}\right) \\
& -B_{1} a_{21}(t)\left(x_{1}-\tilde{x}_{1}\right)-B_{1} a_{22}(t)\left(y_{1}-\tilde{y}_{1}\right)+B_{1} D_{2}(t)\left(\frac{y_{2}}{y_{1}}-\frac{\tilde{y}_{2}}{\tilde{y}_{1}}\right) \\
& -B_{2} b_{21}(t)\left(x_{2}-\tilde{x}_{2}\right)-B_{2} b_{22}(t)\left(y_{2}-\tilde{y}_{2}\right)+B_{2} D_{2}(t)\left(\frac{y_{1}}{y_{2}}-\frac{\tilde{y}_{1}}{\tilde{y}_{2}}\right) \\
\leq & -\left(a_{11}^{L}+a_{21}^{L}\right)\left|x_{1}-\tilde{x}_{1}\right|-\left(b_{11}^{L}+b_{21}^{L}\right)\left|x_{2}-\tilde{x}_{2}\right| \\
- & \left(a_{12}^{L}+a_{22}^{L}\right)\left|y_{1}-\tilde{y}_{1}\right|-\left(b_{12}^{L}+b_{22}^{L}\right)\left|y_{2}-\tilde{y}_{2}\right|+\sum \frac{\tilde{D}_{i}(t)}{i=1} \\
& \\
& \\
& \\
&
\end{aligned}
$$

where

$$
\begin{aligned}
& \tilde{D}_{1}(t)=\left\{\begin{array}{cl}
D_{1}(t)\left(\frac{x_{1}}{x_{2}}-\frac{\tilde{x}_{1}}{\tilde{x}_{2}}\right) & X_{2}(t)-\tilde{X}_{2}(t) \geq 0 \\
D_{1}(t)\left(\frac{\tilde{x}_{1}}{\tilde{x}_{2}}-\frac{x_{1}}{x_{2}}\right) & X_{2}(t)-\tilde{X}_{2}(t)<0
\end{array}\right. \\
& \tilde{D}_{2}(t)= \begin{cases}D_{1}(t)\left(\frac{x_{2}}{x_{1}}-\frac{\tilde{x}_{2}}{\tilde{x}_{1}}\right) & X_{1}(t)-\tilde{X}_{1}(t) \geq 0 \\
D_{1}(t)\left(\frac{\tilde{x}_{2}}{\tilde{x}_{1}}-\frac{x_{2}}{x_{1}}\right) & X_{1}(t)-\tilde{X}_{1}(t)<0\end{cases} \\
& \tilde{D}_{3}(t)=\left\{\begin{array}{cc}
D_{2}(t)\left(\frac{y_{1}}{y_{2}}-\frac{\tilde{y}_{1}}{\tilde{y}_{2}}\right) & Y_{2}(t)-\tilde{Y}_{2}(t) \geq 0 \\
D_{2}(t)\left(\frac{\tilde{y}_{1}}{\tilde{y}_{2}}-\frac{y_{1}}{y_{2}}\right) & Y_{2}(t)-\tilde{Y}_{2}(t)<0
\end{array}\right. \\
& \tilde{D}_{4}(t)=\left\{\begin{array}{cl}
D_{2}(t)\left(\frac{y_{2}}{y_{1}}-\frac{\tilde{y}_{2}}{\tilde{y}_{1}}\right) & Y_{1}(t)-\tilde{Y}_{1}(t) \geq 0 \\
D_{2}(t)\left(\frac{\tilde{y}_{2}}{\tilde{y}_{1}}-\frac{y_{2}}{y_{1}}\right) & Y_{1}(t)-\tilde{Y}_{1}(t)<0
\end{array}\right.
\end{aligned}
$$

There are the following three cases to consider for $\tilde{D}_{1}(t)$ : 
(i) If $X_{2}(t)>\tilde{X}_{2}(t)$ and $t \geq t^{*}$, then

$$
\tilde{D}_{1}(t) \leq \frac{D_{1}(t)}{x_{2}(t)}\left(x_{1}(t)-\tilde{x}_{1}(t)\right) \leq \frac{D_{1}^{M}}{x_{2}^{L}}\left|x_{1}(t)-\tilde{x}_{1}(t)\right|
$$

(ii) If $X_{2}(t)<\tilde{X}_{2}(t)$ and $t \geq t^{*}$, then

$$
\tilde{D}_{1}(t) \leq \frac{D_{1}(t)}{\tilde{x}_{2}(t)}\left(\tilde{x}_{1}(t)-x_{1}(t)\right) \leq \frac{D_{1}^{M}}{x_{2}^{L}}\left|x_{1}(t)-\tilde{x}_{1}(t)\right|
$$

(iii) If $X_{2}(t)=\tilde{X}_{2}(t)$, similar to the above analysis, we can get the same result as (i)and(ii). From (i)-(iii), we have

$$
\tilde{D}_{1}(t) \leq \frac{D_{1}^{M}}{x_{2}^{L}}\left|x_{1}(t)-\tilde{x}_{1}(t)\right| \quad \text { for } \quad t \geq t^{*}
$$

Considering $\tilde{D}_{2}(t), \tilde{D}_{3}(t), \tilde{D}_{4}(t)$ in the same way we can obtain

$\tilde{D}_{2}(t) \leq \frac{D_{1}^{M}}{x_{1}^{L}}\left|x_{2}(t)-\tilde{x}_{2}(t)\right|, \quad \tilde{D}_{3}(t) \leq \frac{D_{2}^{M}}{y_{2}^{L}}\left|y_{1}(t)-\tilde{y}_{1}(t)\right|, \quad \tilde{D}_{4}(t) \leq \frac{D_{2}^{M}}{y_{1}^{L}}\left|y_{2}(t)-\tilde{y}_{2}(t)\right|$

for $t \geq t^{*}$. It then yields that

$$
\begin{aligned}
D^{+} V(t) \leq & -\left(a_{11}^{L}+a_{21}^{L}-\frac{D_{1}^{M}}{x_{2}^{L}}\right)\left|x_{1}-\tilde{x}_{1}\right|-\left(b_{11}^{L}+b_{21}^{L}-\frac{D_{1}^{M}}{x_{1}^{L}}\right)\left|x_{2}-\tilde{x}_{2}\right| \\
& -\left(a_{12}^{L}+a_{22}^{L}-\frac{D_{2}^{M}}{y_{2}^{L}}\right)\left|y_{1}-\tilde{y}_{1}\right|-\left(b_{12}^{L}+b_{22}^{L}-\frac{D_{2}^{M}}{y_{1}^{L}}\right)\left|y_{2}-\tilde{y}_{2}\right|
\end{aligned}
$$

According to the condition of Theorem 3.1, now we let

$$
\begin{aligned}
& P_{1}:=a_{11}^{L}+a_{21}^{L}-\frac{D_{1}^{M}}{x_{2}^{L}}, \quad P_{2}:=b_{11}^{L}+b_{21}^{L}-\frac{D_{1}^{M}}{x_{1}^{L}}, \\
& P_{3}:=a_{12}^{L}+a_{22}^{L}-\frac{D_{2}^{M}}{y_{2}^{L}}, \quad P_{4}:=b_{12}^{L}+b_{22}^{L}-\frac{D_{2}^{M}}{y_{1}^{L}},
\end{aligned}
$$

and $\eta=\min \left\{P_{1}, P_{2}, P_{3}, P_{4}\right\}>0$ then we get that

$$
D^{+} V(t) \leq-\eta\left(\sum_{i=1}^{2}\left|x_{i}-\tilde{x}_{i}\right|+\sum_{i=1}^{2}\left|y_{i}-\tilde{y}_{i}\right|\right)
$$

By Mean Value Theorem,we have following formula:

$$
\begin{aligned}
& \left|x_{i}-\tilde{x}_{i}\right|=\left|e^{X_{i}}-e^{\tilde{X}_{i}}\right|=\zeta_{i}(t)\left|X_{i}-\tilde{X}_{i}\right| \geq x_{i}^{L}\left|X_{i}-\tilde{X}_{i}\right| \\
& \left|y_{i}-\tilde{y}_{i}\right|=\left|e^{Y_{i}}-e^{\tilde{Y}_{i}}\right|=\xi_{i}(t)\left|Y_{i}-\tilde{Y}_{i}\right| \geq y_{i}^{L}\left|Y_{i}-\tilde{Y}_{i}\right|, \quad(i=1,2)
\end{aligned}
$$


where $\zeta_{i}(t) \in\left(x_{i}(t), \tilde{x}_{i}(t)\right)(i=1,2)$ and $\xi_{i}(t) \in\left(y_{i}(t), \tilde{y}_{i}(t)\right)(i=1,2)$ respectively, then $\zeta_{i}(t) \in \Omega$ and $\xi_{i}(t) \in \Omega$.

By the above formula and we take $c=\min \left\{x_{1}^{L} \eta, y_{1}^{L} \eta, x_{2}^{L} \eta, y_{2}^{L} \eta\right\}>0$, then we get that

$$
D^{+} V(t) \leq-c\left(\sum_{i=1}^{2}\left|X_{i}(t)-\tilde{X}_{i}(t)\right|+\sum_{i=1}^{2}\left|Y_{i}(t)-\tilde{Y}_{i}(t)\right|\right)=-c V(t)
$$

It means that $V(t)$ satisfies the condition (iii) of Lemma 2.3. By Lemma 2.3, system (1.2) has a unique almost periodic solution $z(t)$ on the region $\Omega$, which is uniformly asymptotically stable on compact set $\Omega$. Since $\Omega$ is the ultimately bounded region and compact set of system (1.2), hence we get that the solution $z(t)$ is ultimately bounded on $\Omega$, therefore when the conditions of Lemma 2.3 hold, almost periodic solution $z(t)$ is uniformly asymptotically stable. It shows that system (1.2) has a unique almost periodic solution, which is uniformly asymptotically stable. This completes the proof.

\section{Global attractivity}

In this section, we will derive some sufficient conditions for the globally attractivity of system (1.2).

Theorem 4.1. If the system (1.2) satisfies all the conditions of Theorem 3.1, then the unique almost periodic solution of the system (1.2) is globally attractive.

Proof. Let $z(t)=\left(x_{1}(t), x_{2}(t), y_{1}(t), y_{2}(t)\right)$ be a definitive almost periodic solution of the system (1.2), $\tilde{z}(t)=\left(\tilde{x}_{1}(t), \tilde{x}_{2}(t), \tilde{y}_{1}(t), \tilde{y}_{2}(t)\right)$ be any solution of the system (1.2).

Construct the same Liapunov function as defined in the proof of Theorem 3.1,

$$
V(t)=V(t, Z(t), \tilde{Z}(t))=\sum_{i=1}^{2}\left|X_{i}(t)-\tilde{X}_{i}(t)\right|+\sum_{i=1}^{2}\left|Y_{i}(t)-\tilde{Y}_{i}(t)\right|
$$

Integrating both sides of (3.1) from 0 to t, we can derive

$$
V(t)+\eta \int_{0}^{t}\left(\sum_{i=1}^{2}\left|x_{i}(s)-\tilde{x}_{i}(s)\right|+\sum_{i=1}^{2}\left|y_{i}(s)-\tilde{y}_{i}(s)\right|\right) d s \leq V(0)
$$

The expression (4.2) shows that

$$
0 \leq V(t) \leq V(0)=\sum_{i=1}^{2}\left|X_{i}(0)-\tilde{X}_{i}(0)\right|+\sum_{i=1}^{2}\left|Y_{i}(0)-\tilde{Y}_{i}(0)\right|<+\infty, \quad t \geq 0
$$


and

$$
\int_{0}^{t}\left(\sum_{i=1}^{2}\left|x_{i}(s)-\tilde{x}_{i}(s)\right|+\sum_{i=1}^{2}\left|y_{i}(s)-\tilde{y}_{i}(s)\right|\right) d s \leq \frac{V(0)}{\eta}<+\infty, \quad t \geq 0
$$

The expression (4.4) implies that

$$
\sum_{i=1}^{2}\left|x_{i}(s)-\tilde{x}_{i}(s)\right|+\sum_{i=1}^{2}\left|y_{i}(s)-\tilde{y}_{i}(s)\right| \in L^{1}[0,+\infty)
$$

Obviously $x_{i}(t)$ and $y_{i}(t)(i=1,2)$ are uniformly bounded, so $X_{i}(t)$ and $Y_{i}(t)(i=1,2)$ are also uniformly bounded. In addition, by (4.1)-(4.3), we can know that $\tilde{X}_{i}(t)$ and $\tilde{Y}_{i}(t)(i=1,2)$ are uniformly bounded, so $\tilde{x}_{i}(t)$ and $\tilde{y}_{i}(t)(i=1,2)$ are also uniformly bounded. Combining this fact with the system (1.2), we have $\dot{x}_{i}, \dot{y}_{i}, \dot{\tilde{x}}_{i}, \dot{\tilde{y}}_{i}(i=1,2)$ are uniformly bounded. Therefore we can easily check $\left[x_{i}(t)-\tilde{x}_{i}(t)\right]$ and $\left[y_{i}(t)-\right.$ $\left.\tilde{y}_{i}(t)\right](i=1,2)$ and their derivatives remain bounded on $[0,+\infty)$. As a consequence $\sum_{i=1}^{2}\left|x_{i}(t)-\tilde{x}_{i}(t)\right|+\sum_{i=1}^{2}\left|y_{i}(t)-\tilde{y}_{i}(t)\right|$ is uniformly continuous on $[0,+\infty)$. From the expression (4.5), it follows that $\sum_{i=1}^{2}\left|x_{i}(t)-\tilde{x}_{i}(t)\right|+\sum_{i=1}^{2}\left|y_{i}(t)-\tilde{y}_{i}(t)\right|$ is integrable on $[0,+\infty)$. By Lemma 2.4 , it follows that

$$
\lim _{t \rightarrow \infty}\left(\sum_{i=1}^{2}\left|x_{i}(t)-\tilde{x}_{i}(t)\right|+\sum_{i=1}^{2}\left|y_{i}(t)-\tilde{y}_{i}(t)\right|\right)=0
$$

Hence

$$
\lim _{t \rightarrow \infty}\left|x_{i}(t)-\tilde{x}_{i}(t)\right|=0, \quad \lim _{t \rightarrow \infty}\left|y_{i}(t)-\tilde{y}_{i}(t)\right|=0 \quad(i=1,2)
$$

This result implies that the unique almost periodic solution of system (1.2) is stable and attracts all positive solution of system (1.2). This completes the proof. 


\section{One example}

Example. we consider the following system

$$
\begin{aligned}
\dot{x}_{1}(t)= & x_{1}(t)\left(5+0.5(\sin (\sqrt{2} t)+\sin (t))-(2.5+0.5(\cos (\sqrt{2} t)+\cos (t))) x_{1}(t)\right. \\
& \left.-(2.2+0.3(\sin (\sqrt{2} t)+\sin (t))) y_{1}(t)\right) \\
& +(1+0.1(\cos (\sqrt{2} t)+\cos (t)))\left(x_{2}(t)-x_{1}(t)\right), \\
\dot{y}_{1}(t)= & y_{1}(t)\left(5+0.4(\sin (\sqrt{2} t)+\sin (t))-(2.25+0.6(\cos (\sqrt{2} t)+\cos (t))) x_{1}(t)\right. \\
& \left.-(2.4+0.4(\sin (\sqrt{2} t)+\sin (t))) y_{1}(t)\right) \\
& +(1+0.2(\sin (\sqrt{2} t)+\sin (t)))\left(y_{2}(t)-y_{1}(t)\right), \\
\dot{x}_{2}(t)= & x_{2}(t)\left(4+0.5(\cos (\sqrt{2} t)+\cos (t))-(2.4+0.7(\sin (\sqrt{2} t)+\sin (t))) x_{2}(t)\right. \\
& \left.-(2.3+0.5(\cos (\sqrt{2} t)+\cos (t))) y_{2}(t)\right) \\
& +(1+0.1(\cos (\sqrt{2} t)+\cos (t)))\left(x_{2}(t)-x_{1}(t)\right), \\
\dot{y}_{2}(t)= & y_{2}(t)\left(4+0.3(\cos (\sqrt{2} t)+\cos (t))-(2.3+0.5(\sin (\sqrt{2} t)+\sin (t))) x_{2}(t)\right. \\
& \left.-(2.5+0.3(\cos (\sqrt{2} t)+\cos (t))) y_{2}(t)\right) \\
& +(1+0.2(\sin (\sqrt{2} t)+\sin (t)))\left(y_{1}(t)-y_{2}(t)\right),
\end{aligned}
$$

It is easy to verify all the conditions required in Theorem 3.1 and Theorem 4.1 are satisfied. Then the system (5.1) has a unique almost periodic solution and which is asymptotically stable and globally attractive.

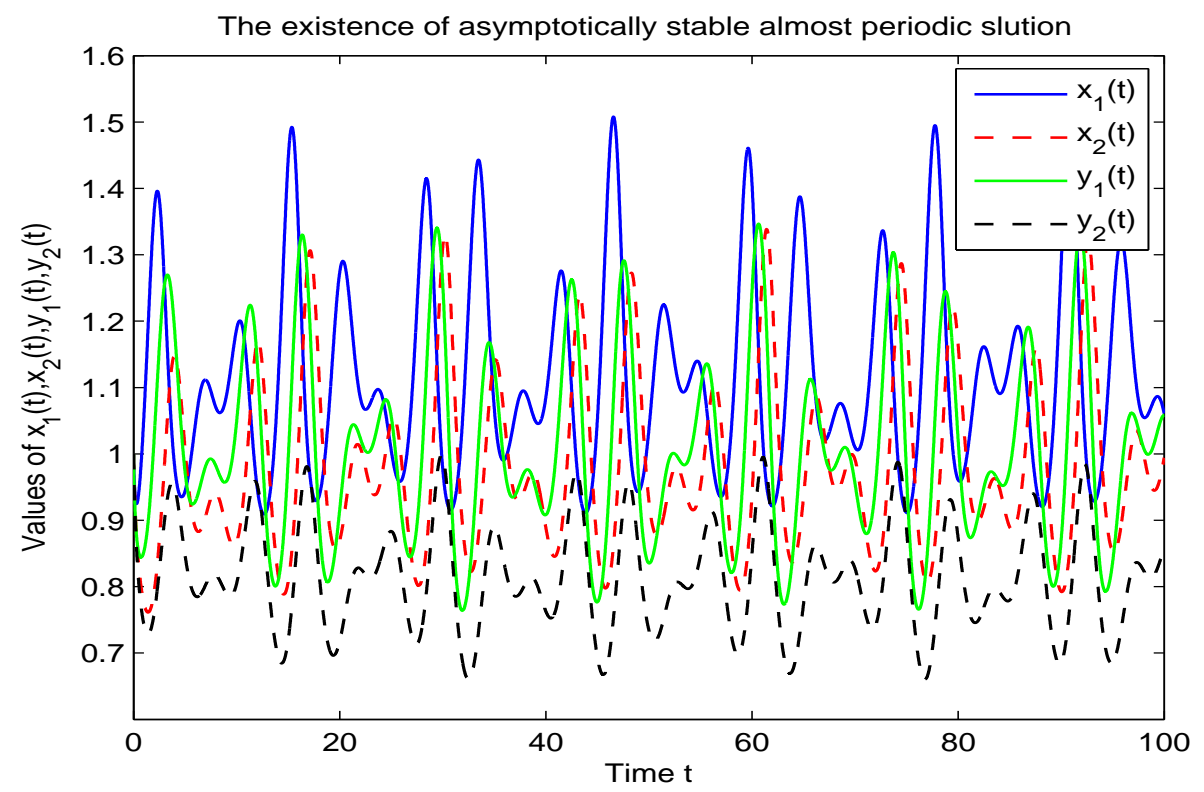

Fig. 1. The existence of almost periodic solutions for system $(5.1)$. Here, we take the initial value $x_{0}=\left(x_{10}, x_{20}, y_{10}, y_{20}\right)=(1,1,1,1)$. 

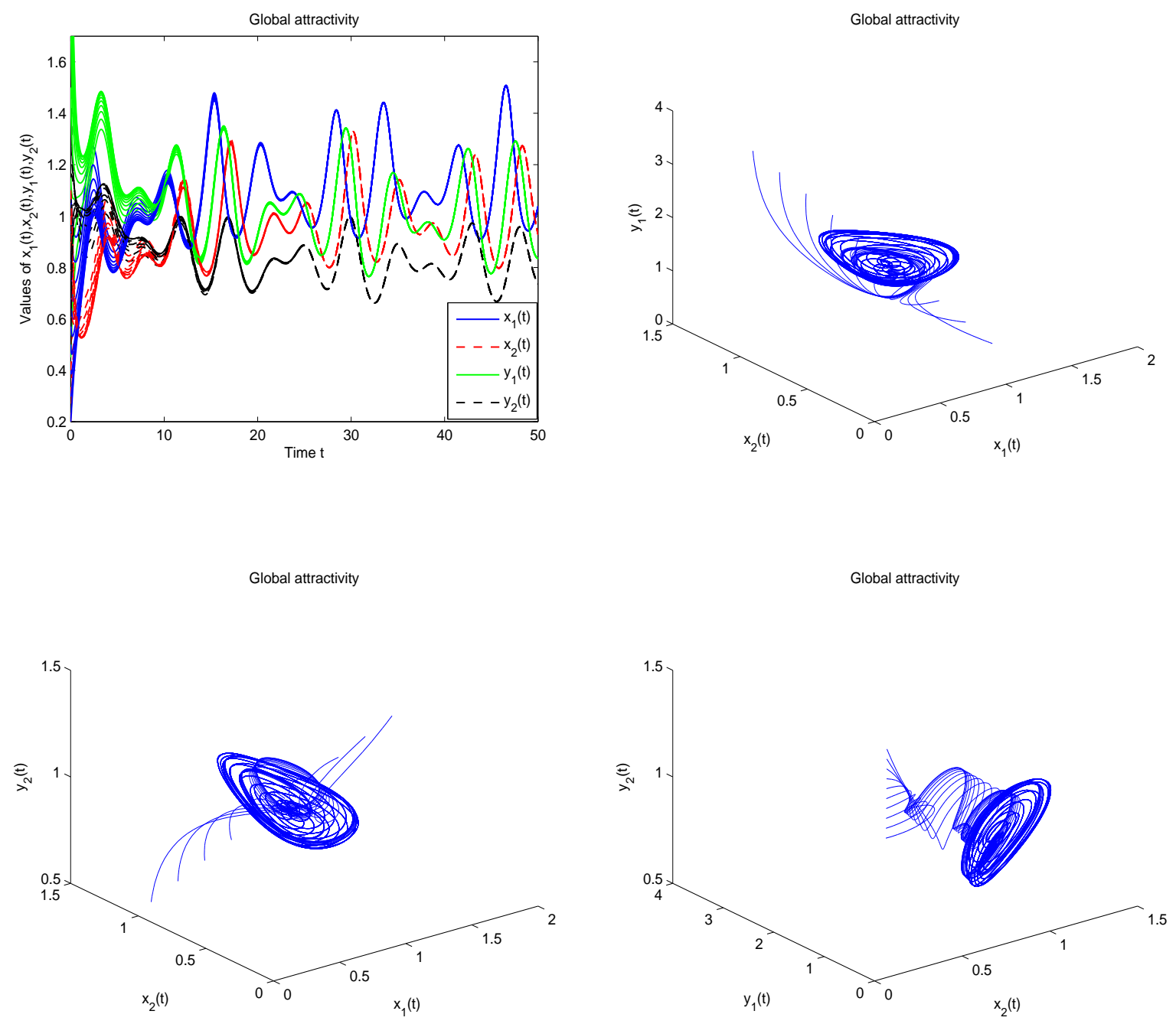

Fig. 2. Global attractivity of almost periodic solutions for system (5.1). Here, we take different initial values.

Figure 1 shows that the system (5.1) converges to a almost periodic solution. Figure 2 shows that the almost periodic solution of system (5.1) is globally attractive.

\section{References}

[1] F. Chen, Persistence and global stability for nonautonomous cooperative system with diffusion and time delay, Acta Scientiarum Naturalium Universitatis Pekinensis. 39(2003), 22-28.

[2] X. Ding, F. Wang, Positive periodic solution for a semi-ratio-dependent predator-prey system with diffusion and time delays, Nonl. Anal.: RWA. 9(2008), 239-249. 
[3] L. Dong, L. Chen, P. Shi, Periodic solutions for a two-species nonautonomous competition system with diffusion and impulses, Chaos, Solitons and Fractals. 32(2007), 1916-1926.

[4] K. Gopalasamy, Stability and Oscillation in Delay Equation of Population Dynamics, Kluwer Academic Publishers, Dordrecht, 1992.

[5] K. Kishimoto, Coexistence of any number of species in the Lotka-Volterra competition system over two-patches, Theor. Popul. Biol. 38(1990), 149-158.

[6] Y. Liang, L. Li, L. Chen, Almost periodic solutions for Lotka-Volterra systems with delays , Commun Nonlinear Sci Numer Simulat. 14(2009), 3660-3669.

[7] C. Liu, L. Chen , Periodic solution and global stability for nonautonomous cooperative Lotka-Volterra diffusion system, Journal of LanZhou University (Natural Science). 33(1997), 33-37.

[8] Z. Liu, S. Zhong, Permanence and extinction analysis for a delayed periodic predator-prey system with Holling type II response function and diffusion, Appl. Math. Comput. 216(2010), 3002-3015.

[9] X. Meng, L. Chen, Periodic solution and almost periodic solution for a nonautonomous Lotka-Volterra dispersal system with infinite delay, J.Math. Anal. Appl.339(2008), 125-145.

[10] X. Song, L.Chen, Uniform persistence and global attractivity for nonautonomous competitive systems with dispersion, J. Syst.Sci. Complex. 15(2002), 307-314.

[11] F. Wei, Y. Lin, L. Que, Y. Chen, Y. Wu, Y. Xue, Periodic solution and global stability for a nonautonomous competitive Lotka-Volterra diffusion system, Appl. Math.Comput. 216(2010), 3097-3104.

[12] F. Wei, K. Wang, Almost periodic solution and stability for nonautonomous cooperative Lotka-Volterra diffusion system, SongLiao Journal (Natural Science Edition). 3(2002), 1-4.

[13] F. Wei, K. Wang, Global stability and asymptotically periodic solution for nonautonomous cooperative Lotka-Volterra diffusion system, Appl. Math.Comput. 182(2006),161-165. 
[14] F. Wei, S. Wang, Almost periodic solution and global stability for cooperative LV diffusion system, J. Mathematical Research and Exposition. 30(2010), 11081116.

[15] G. Zeng, L. Chen, Persistence and periodic orbits for two-species nonautonomous diffusion Lotka-Volterra models, Math. Comput. Modelling. 20(1994), 69-80.

[16] J. Zhang, L. Chen, Permanence and global stability for two-species cooperative system with delays intwo-patch environment, Math. Comput. Modelling. 23(1996), 17-27.

[17] J. Zhang, L. Chen, X. Chen, Persistence and global stability for two-species nonautonomous competition Lotka-Volterra patch-system with time delay, Nonl. Anal. 37(1999), 1019-1028.

[18] Z. Zhang, Z. Wang, Periodic solution for a two-species nonautonomous competition Lotka-olterra patch system with time delay, J.Math. Anal. Appl. 265(2002), 38-48.

[19] X. Zhou, X. Shi, X. Song, Analysis of nonautonomous predator-prey model with nonlinear diffusion and time delay, Applied Mathematics and Computation. 196(2008),129-136. 